\title{
Prevalence of Airflow Limitation Defined by Pre- and Post- Bronchodilator Spirometry in a Community-Based Health Checkup: The Hisayama Study
}

\author{
Satoru Fukuyama, ${ }^{1, *}$ Koichiro Matsumoto, ${ }^{1, *}$ Yasuko Kaneko, ${ }^{1}$ Keiko Kan-o, ${ }^{1}$ \\ Naotaka Noda, ${ }^{1}$ Yukari Tajiri-Asai, ${ }^{1}$ Takako Nakano, ${ }^{1}$ Yumiko Ishii, ${ }^{1}$ \\ Yutaka Kiyohara, ${ }^{2}$ Yoichi Nakanishi, ${ }^{1}$ Hiromasa Inoue ${ }^{1,3}$ and \\ the Hisayama Pulmonary Physiology Study Group \\ ${ }^{1}$ Research Institute for Diseases of the Chest, Graduate School of Medical Sciences, Kyushu University, Fukuoka, \\ Fukuoka, Japan \\ ${ }^{2}$ Department of Environmental Medicine, Graduate School of Medical Sciences, Kyushu University, Fukuoka, \\ Fukuoka, Japan \\ ${ }^{3}$ Department of Pulmonary Medicine, Graduate School of Medical and Dental Sciences, Kagoshima University, \\ Kagoshima, Kagoshima, Japan
}

\begin{abstract}
Spirometry in health checkup may contribute to early diagnosis of chronic obstructive pulmonary disease (COPD) and asthma. Although post-bronchodilator airflow limitation is essential for definite diagnosis of COPD and post-bronchodilator normalization of airflow is suggestive of asthma, this test has not been prevailed in health checkup. The objective of this study was to estimate the prevalence of airflow limitation defined by pre- and post-bronchodilator spirometry in health checkup. Post-bronchodilator spirometry was conducted for participants with airflow limitation in a town-wide health checkup for residents aged 40 years and older in Hisayama, a town in the western part of Japan. The prevalence of pre- and postbronchodilator airway limitation defined by $\mathrm{FEV}_{1} / \mathrm{FVC}<70 \%$ were estimated. A total of 2,232 participants underwent pre-bronchodilator spirometry. In males, the age of current smokers was significantly younger than those of never smokers and former smokers. In females, the ages of current- and former smokers were significantly younger than never smokers. The values of $\% \mathrm{FEV}_{1}$ and $\% \mathrm{FVC}$ in current smokers were significantly lower than those in former smokers and never smokers. Two hundred sixty nine subjects, $85 \%$ of total subjects with a pre-bronchodilator $\mathrm{FEV}_{1} / \mathrm{FVC}<70 \%$, completed post-bronchodilator spirometry. The prevalence of pre-bronchodilator airflow limitation was $14.6 \%$ in males and $13.7 \%$ in females, and the prevalence of post-bronchodilator airway limitation was $8.7 \%$ and $8.7 \%$, respectively. Post-bronchodilator spirometry in health checkup would reduce the number of subjects with probable COPD to two-third. Recommendation for those examinees to take further evaluations may pave the way for early intervention.
\end{abstract}

Keywords: asthma; bronchodilator; chronic obstructive pulmonary diseases; prevalence; spirometry

Tohoku J. Exp. Med., 2016 February, 238 (2), 179-184. (C) 2016 Tohoku University Medical Press

\section{Introduction}

Chronic obstructive pulmonary disease (COPD) is a major challenge to public health and socioeconomic burden particularly on countries of accelerated aging with a low birth rate including Japan (Bustacchini et al. 2011). Asthma is also a major disease involving a wide range of generation from infancy to elderly. Given that COPD and asthma is revealed by airway limitation in spirometry, involvement of spirometry to community-based health checkup may become an "easy access" improving early diagnosis. In spirometry, airflow can be assessed as forced expiratory volume in one second $\left(\mathrm{FEV}_{1}\right)$, while lung volume can be assessed as forced vital capacity (FVC). $\mathrm{FEV}_{1} / \mathrm{FVC}$ lower than $70 \%$ indicates airflow limitation. Importantly, postbronchodilator spirometry is essential for definite diagnosis of COPD to demonstrate airway limitation that is not fully reversible (Global Initiative for Chronic Obstructive Lung Disease 2014). Normalization of airflow by bronchodilator may indicate possible asthma. This test has not been prevailed in health checkups and the examinees with airflow limitation are recommended to have further evaluation

Received November 26, 2015; revised and accepted January 13, 2016. Published online February 16, 2016; doi: 10.1620/tjem.238.179.

*These two authors contributed equally to this work.

Correspondence: Koichiro Matsumoto, Research Institute for Diseases of the Chest, Graduate School of Medical Sciences, Kyushu University, 3-1-1 Maidashi, Higashi-ku, Fukuoka, Fukuoka 812-8582, Japan.

e-mail: koichi@kokyu.med.kyushu-u.ac.jp 
including post-bronchodilator spirometry at pulmonary physician's clinics. However, a substantial proportion of examinees default further evaluation, which results in a useless cost of health checkup. In this regard, completion of post-bronchodilator spirometry at the health checkup may reduce the default evaluation and then improve costeffectiveness. The relevance of post-bronchodilator spirometry in health checkup should be investigated.

The Hisayama Study is an ongoing population-based epidemiologic study for cardiovascular diseases and smoking-related diseases and their risk factors in the community in Hisayama, a town in the western part of Japan (Hata et al. 2013; Toyoda et al. 2013). The population of the town is approximately 8,300 and has been stable for more than the last 40 years. The age and occupational distributions of the Hisayama population have been almost identical to those of Japan as a whole from the 1960s to the present, based on data from the nation census (The Statistics Bureau of Japan 2010). In 2009, post-bronchodilator spirometry was conducted for participants with airflow limitation in a townwide health checkup for residents aged 40 years and older. The objective of this study was to estimate the prevalence of airflow limitation defined by pre- and post-bronchodilator spirometry in a community-based health checkup.

\section{Materials and Methods}

Study population, spirometry, and ethics

All registered residents aged 40 years and older were invited to participate in a town-wide health checkup including spirometry from June to August in 2009. Subjects completed a self-reported questionnaire on cigarette smoking. Individual height and weight were measured at the beginning of health checkup. Subjects with the following conditions were excluded from spirometry: current chest pain; had recent surgery of the eye, chest or the abdomen; had a recent history of pneumothorax; had a past history of lung resection, or refusal. Spirometry was performed under guidelines of the Japanese Respiratory Society (The Japanese Respiratory Society 2004) using a CHESTGRAPH HI-105 spirometer (Chest MI, Inc., Tokyo, Japan). Up to four tests were used to obtain satisfactory flow-volume loop recordings. Pulmonary physicians assessed the results by visual inspection to the loop recordings and excluded those without at least two satisfactory tests. Subjects with poor maneuver were excluded. The largest $\mathrm{FVC}$ and the largest $\mathrm{FEV}_{1}$ from all acceptable maneuvers were selected. Subjects who had $\mathrm{FEV}_{1} / \mathrm{FVC}<70 \%$ were eligible for post-bronchodilator testing, in which spirometry was performed 15 min after inhalation of salbutamol (GlaxoSmithKline, Tokyo, Japan) via a metered-dose inhaler with a spacer, according to the procedure recommended (Pellegrino et al. 2005). Subjects with the following conditions were excluded from post-bronchodilator spirometry: had a recent history of ischemic heart attack, unstable arrhythmia requiring medications, or refusal. Subjects were also excluded if they had already taken a $\beta_{2}$-adrenergic bronchodilator to avoid exceeding doses, of if they had had a previous history of adverse reaction to salbutamol. The written informed consent for potential risk and benefit to use salbutamol inhaler was obtained from all subjects with postbronchodilator spirometry. Subjects who had post-bronchodilator $\mathrm{FEV}_{1} / \mathrm{FVC}<70 \%$ were recommended to have further evaluations in the clinics of pulmonary medicine and to have adequate pharmacotherapy if necessary. Reference values for spirometric measures predicted were derived from Japanese criteria updated on 2014 (Kubota et al. 2014). The study was conducted according to the Declaration of Helsinki and approved by the Institutional Review Board for Clinical Research of Kyushu University (Approved \#: 19-15 and 21-37), and written informed consent for medical research was obtained from the subjects.

\section{Smoking status}

We defined "never smokers" those individuals who declared that they never smoked before the study, "current smokers" those who declared active smoking, and "former smokers" those who declared that they quitted smoking before the study. Those who declared that they quitted smoking just before $(<1$ month) the study were classified into current smokers. Pack-years were calculated as the number of packs of cigarettes smoked per day times how many years the participants smoked.

\section{Statistical analysis}

Results are presented as the mean \pm standard deviation (SD). Differences in categorical variables were assessed by chi-square tests. Differences in continuous variables with normal distribution, such as age, the body mass index (BMI), $\mathrm{FEV}_{1}, \mathrm{FVC}, \mathrm{FEV}_{1} / \mathrm{FVC}$, and $\% \mathrm{FEV}_{1} / \mathrm{FVC}$, were assessed by means of analyses of variance with post hoc Tukey-Kramer's HSD tests. Differences in skewed continuous variable, such as pack-years, $\% \mathrm{FEV}_{1}$, and $\mathrm{FVC}$, were assessed by Kruskal-Wallis tests with post hoc Mann-Whitney $U$-tests. P values of less than 0.05 were considered to indicate statistical significance.

\section{Results}

\section{Eligibility of participants}

Flow diagram of the study population is shown in Fig. 1. A total of 2,251 subjects participated in the health checkup over the period from July to August 2009. All participants were Asians. Nineteen subjects were excluded from the study due to safety reasons, refusal or poor maneuver. Of the 2,232 subjects, 315 had a pre-bronchodilator $\mathrm{FEV}_{1} / \mathrm{FVC}$ of less than $70 \%$ and 46 were excluded from post-bronchodilator spirometry due to safety reasons, refusal, or poor inhalation maneuver. Remaining 269 subjects, $85 \%$ of total subjects with a pre-bronchodilator $\mathrm{FEV}_{1} /$ FVC of less than $70 \%$, completed post-bronchodilator spirometry.

\section{Characteristics of the participants}

The baseline characteristics of the 2,232 subjects eligible for the final analyses are shown in Table 1. In males, the age of current smokers was significantly younger than those of never smokers $(\mathrm{p}<0.001)$ and former smokers $(\mathrm{p}$ $<0.001)$. The age of former smokers was significantly older than that of never smokers $(\mathrm{p}<0.001)$. The BMI of current smokers was significantly lower than those of never smokers $(\mathrm{p}<0.05)$ and former smokers $(\mathrm{p}<0.05)$, while there was no significant difference between those of former smokers and never smokers. The pack-year of current smokers was significantly higher than that of former smok- 


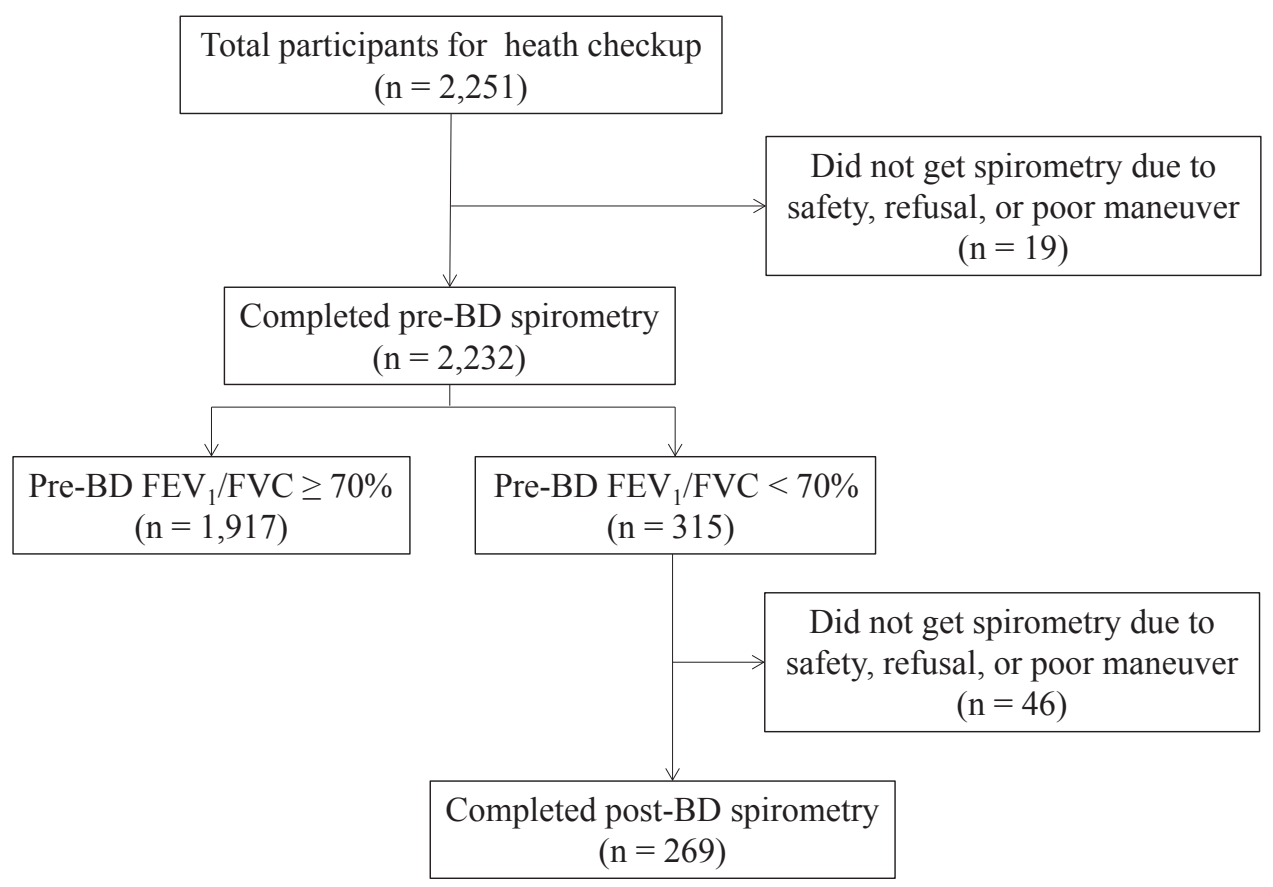

Fig. 1. Flow diagram of the study population.

$\mathrm{BD}$, bronchodilator; $\mathrm{FEV}_{1}$, forced expiratory volume in the one second; $\mathrm{FVC}$, forced vital capacity.

ers $(p<0.001)$. In females, the ages of current smokers and former smokers were significantly younger than never smokers $(\mathrm{p}<0.001, \mathrm{p}<0.001$, respectively), while there was no significant difference between those of current smokers and former smokers. The BMI was not significantly different among never-, former-, and current smokers. The pack-year of current smokers was significantly higher than that of former smokers $(\mathrm{p}<0.001)$.

\section{Pre-bronchodilator spirometric analyses}

The pre-bronchodilator spirometric measures are also shown in Table 1. Both in males and females, the values of $\mathrm{FEV}_{1}$ and $\mathrm{FVC}$ and the $\mathrm{FEV}_{1} / \mathrm{FVC}$ ratio were not significantly different among never smokers, former smokers, and current smokers. In males, the value of $\% \mathrm{FEV}_{1}$ in current smokers was significantly lower than those in former smokers $(p<0.001)$ and never smokers $(p<0.05)$. The value of $\% \mathrm{FVC}$ in current smokers was also significantly lower than those in former smokers $(\mathrm{p}<0.05)$ and never smokers $(\mathrm{p}<$ $0.05)$. The value of $\% \mathrm{FEV}_{1} / \mathrm{FVC}$ in current smokers was significantly lower than that in former smokers $(\mathrm{p}<0.01)$, but it was similar to that in never smokers. The values of $\% \mathrm{FEV}_{1}, \% \mathrm{FVC}$, and $\% \mathrm{FEV}_{1} / \mathrm{FVC}$ did not significantly differ between former smokers and never smokers. In females, the values of $\% \mathrm{FEV}_{1}$ in current smokers and former smokers were significantly lower than that in never smokers ( $p<0.01, p<0.001$, respectively). The values of $\% \mathrm{FVC}$ in current smokers and former smokers were also significantly lower than that in never smokers $(\mathrm{p}<0.01, \mathrm{p}<$ 0.001 , respectively). There was no significant difference in the values of $\% \mathrm{FEV}_{1}$ or $\% \mathrm{FVC}$ between former smokers and current smokers. The values of $\% \mathrm{FEV}_{1} / \mathrm{FVC}$ were not significantly different among never smokers, former smokers, and current smokers in females.

Prevalence of pre- and post-bronchodilator airflow limitation The prevalence of airflow limitation is shown in Table 2. In male subjects, the prevalence of airflow limitation defined by pre-bronchodilator $\mathrm{FEV}_{1} / \mathrm{FVC}$ was $17.3 \%$ in never smokers, $13.6 \%$ in former smokers, $14.4 \%$ in current smokers, and $14.6 \%$ in total. There was no significant difference in the prevalence among never-, former- and current smokers $\left(\chi^{2}=1.53, \mathrm{p}=0.4653\right)$. Applying the same criteria to post-bronchodilator $\mathrm{FEV}_{1} / \mathrm{FVC}$ results, the prevalence of airflow limitation that is not fully reversible was $9.4 \%$ in never smokers, $8.5 \%$ in former smokers, $8.5 \%$ in current smokers, and $8.7 \%$ in total. Again, there was no significant difference in the prevalence among never smokers, former smokers and current smokers $\left(\chi^{2}=0.22, p=0.8957\right)$. In female subjects, the prevalence of airflow limitation defined by pre-bronchodilator $\mathrm{FEV}_{1} / \mathrm{FVC}$ was $13.4 \%$ in never smokers, $19.7 \%$ in former smokers, $10.5 \%$ in current smokers, and $13.7 \%$ in total. There was no significant difference in the prevalence among never-, former- and current smokers $\left(\chi^{2}=3.22, p=0.2002\right)$. Applying the same criteria to post-bronchodilator $\mathrm{FEV}_{1} / \mathrm{FVC}$ results, the prevalence of airflow limitation was $8.6 \%$ in never smokers, $10.4 \%$ in former smokers, $8.7 \%$ in current smokers, and $8.7 \%$ in total. There was no significant difference among never smokers, former smokers and current smokers $\left(\chi^{2}=0.51, \mathrm{p}\right.$ $=0.7759$ ). 
Table 1. The subjects' characteristics and the pre-bronchodilator spirometric measures.

\begin{tabular}{|c|c|c|c|c|c|c|}
\hline \multirow{2}{*}{$\frac{\text { Sex }}{\text { Smoking status }}$} & \multicolumn{3}{|c|}{ Males $(\mathrm{n}=951)$} & \multicolumn{3}{|c|}{ Females $(\mathrm{n}=1,281)$} \\
\hline & $\begin{array}{c}\text { NS } \\
\mathrm{n}=190\end{array}$ & $\begin{array}{c}\text { FS } \\
\mathrm{n}=491\end{array}$ & $\begin{array}{c}\text { CS } \\
n=270\end{array}$ & $\begin{array}{c}\text { NS } \\
\mathrm{n}=1,138\end{array}$ & $\begin{array}{c}\text { FS } \\
\mathrm{n}=86\end{array}$ & $\begin{array}{c}\mathrm{CS} \\
\mathrm{n}=57\end{array}$ \\
\hline Age (years) & $63.9 \pm 11.2$ & $67.4 \pm 10.4 *$ & $58.5 \pm 10.5^{*, \dagger}$ & $64.3 \pm 11.2$ & $57.2 \pm 11.2 *$ & $55.9 \pm 9.8^{*}$ \\
\hline BMI $\left(\mathrm{kg} / \mathrm{m}^{2}\right)$ & $23.5 \pm 2.9$ & $23.4 \pm 2.9$ & $22.8 \pm 2.9^{*, \dagger}$ & $22.8 \pm 3.6$ & $23.1 \pm 3.8$ & $22.3 \pm 3.9$ \\
\hline Pack-years & & $31.8 \pm 22.9$ & $39.6 \pm 20.0^{\dagger}$ & & $9.9 \pm 13.5$ & $22.0 \pm 11.7^{\dagger}$ \\
\hline $\mathrm{FEV}_{1}(\mathrm{~L})$ & $2.18 \pm 0.65$ & $2.18 \pm 0.62$ & $2.17 \pm 0.63$ & $2.16 \pm 0.64$ & $2.01 \pm 0.61$ & $2.05 \pm 0.53$ \\
\hline$\% \mathrm{FEV}_{1}(\%)$ & $79.8 \pm 28.4$ & $81.1 \pm 26.5$ & $73.2 \pm 24.7^{*, \dagger}$ & $111.0 \pm 40.3$ & $93.1 \pm 32.0^{*}$ & $93.0 \pm 25.5^{*}$ \\
\hline FVC (L) & $2.88 \pm 0.78$ & $2.86 \pm 0.75$ & $2.86 \pm 0.79$ & $2.86 \pm 0.78$ & $2.67 \pm 0.75$ & $2.69 \pm 0.67$ \\
\hline$\% \mathrm{FVC}(\%)$ & $82.3 \pm 26.8$ & $82.4 \pm 24.6$ & $76.4 \pm 24.2^{*, \dagger}$ & $115.8 \pm 38.6$ & $99.1 \pm 31.3^{*}$ & $98.2 \pm 25.9^{*}$ \\
\hline $\mathrm{FEV}_{1} / \mathrm{FVC}(\%)$ & $75.5 \pm 7.2$ & $76.1 \pm 7.0$ & $75.5 \pm 6.6$ & $75.6 \pm 7.1$ & $75.0 \pm 7.7$ & $75.8 \pm 7.5$ \\
\hline$\% \mathrm{FEV}_{1} / \mathrm{FCV}(\%)$ & $96.3 \pm 9.5$ & $97.9 \pm 9.5$ & $95.2 \pm 8.7^{\dagger}$ & $95.4 \pm 9.3$ & $93.3 \pm 10.0$ & $94.1 \pm 9.0$ \\
\hline
\end{tabular}

Values are expressed as the mean $\pm \mathrm{SD}$.

NS, never smokers; FS, former smokers; CS, current smokers; BMI, body mass index; $\mathrm{FEV}_{1}$, forced expiratory volume in one second; FVC, forced vital capacity.

Pack-years were calculated based on the number of cigarettes smoked per day and the number of years that the subjects had smoked.

$* \mathrm{P}<0.05$, vs. NS

${ }^{\mathrm{P}}<0.05$, vs. FS.

Table 2. The prevalence of airflow limitation defined by $\mathrm{FEV}_{1} / \mathrm{FVC}<70 \%$.

\begin{tabular}{|c|c|c|c|c|c|c|}
\hline \multirow{2}{*}{$\frac{\text { Sex }}{\text { Smoking status }}$} & \multicolumn{3}{|c|}{ Males $(\mathrm{n}=951)$} & \multicolumn{3}{|c|}{ Females $(\mathrm{n}=1,281)$} \\
\hline & NS & FS & $\mathrm{CS}$ & NS & FS & $\mathrm{CS}$ \\
\hline Pre-BD FEV $1 /$ FVC $<70 \% /$ subjects & $33 / 190$ & $67 / 491$ & $39 / 270$ & $153 / 1,138$ & $17 / 86$ & $6 / 57$ \\
\hline Total & & $139 / 951$ & & & $176 / 1,281$ & \\
\hline Post-BD FEV $\mathrm{FVVC}_{1} / \mathrm{F} \% \% /$ subjects & $18 / 27$ & $42 / 57$ & $23 / 36$ & $98 / 132$ & $9 / 12$ & $5 / 5$ \\
\hline Total & & $83 / 120$ & & & $112 / 149$ & \\
\hline Pre-BD prevalence $(\%)$ & 17.3 & 13.6 & 14.4 & 13.4 & 19.7 & 10.5 \\
\hline Total $(\%)$ & & 14.6 & & & 13.7 & \\
\hline Post-BD prevalence $(\%)$ & 9.4 & 8.5 & 8.5 & 8.6 & 10.4 & 8.7 \\
\hline Total $(\%)$ & & 8.7 & & & 8.7 & \\
\hline
\end{tabular}

$\mathrm{FEV}_{1}$, forced expiratory volume in one second; FVC, forced vital capacity; BD, bronchodilator; NS, never smokers; FS, former smokers; CS, current smokers.

\section{Discussion}

In this study, the prevalence of airflow limitation defined by pre-bronchodilator $\mathrm{FEV}_{1} / \mathrm{FVC}<70 \%$ was $14.6 \%$ in males and $13.7 \%$ in females, and the prevalence of post-bronchodilator airway limitation was $8.7 \%$ and $8.7 \%$, respectively. The post-bronchodilator values are consistent with those estimated as the prevalence of COPD in a previous epidemiological study in Japan (Fukuchi et al. 2004). National institutes of USA reported the prevalence of COPD on a nationally representative sample of US adults aged 40-79 years: the prevalence of pre-bronchodilator airway limitation was $24.1 \%$ in males and $16.7 \%$ in females, while post-bronchodilator airway limitation was $11.4 \%$ and $5.4 \%$, respectively (Tilert et al. 2013). These results suggest that post-bronchodilator spirometry would reduce the number of subjects with probable COPD to onethird to two-third in health checkup. Intensive recommendation for those examinees to take further evaluations may improve early intervention and efficacy of health checkup.

Investigators in the UK reported the prevalence of prebronchodilator airflow limitation on a cross-sectional analysis of persons aged 40-95 years in England and Wales: the prevalence was $26.3 \%$ in males and $18.6 \%$ in females (Scholes et al. 2014). Fukuchi et al. (2004) reported the prevalence of pre-bronchodilator airflow limitation on 2,343 subjects aged 40 years and older who were demographically matched to the Japanese general population: the prevalence of airflow limitation was $16.4 \%$ in males and $5.0 \%$ in females. The prevalence of pre-bronchodilator airway limitation in males in the present study was comparable with that in the previous report in Japan but lower than 
those in the USA and the UK, while the prevalence in females was comparable with those in the USA and the UK but markedly higher than that in the previous report in Japan. The discrepant results in females between the studies in Japan might be partly derived from the difference in the study designs that the previous study was based on a population-matched sample while the present study was on a community-based sample. Accumulation of studies with similar design, in addition to studies on workplace-based health checkups, would provide better understanding for this issue.

The present study has several limitations. The study did not intend to discriminate between COPD and asthma. In previous studies, current smoking increased the prevalence of airflow limitation and the incidence of COPD (Kojima et al. 2007; Kohansal et al. 2009), while there is no significant association between smoking and the prevalence of airflow limitation in the present study. The documentation of not-fully reversible airflow limitation is a functional definition of COPD, which, however, does not preclude chronic asthma or asthma with airway remodeling. It is possible that the high prevalence of airflow limitation in never smokers was largely derived from subjects with undiagnosed asthma. In our prior study in Hisayama, $75 \%$ of subjects aged 40 years and older with physician-diagnosed asthma were never smokers (Matsumoto et al. 2015). As another possibility, the composition of age and age-specific factors in the examinees might affect the outcome. The proportion of the aged $4^{\text {th }}$ and $5^{\text {th }}$ decades, belonging to a working population and having high probability of current smoking, was less than that of the aged older, probably due to the inconvenience to have a community-based health checkup. Despite that the number of those middle-aged populations in Hisayama was comparable with that of the aged $6^{\text {th }}$ decade, the participants of health checkup were only one-third to a half of the aged $6^{\text {th }}$ decade. It is possible that the middle-aged participants might have some phenotype that prone to accompany with airflow limitation. With this regard, the mean age of groups (never smokers, former smokers and current smokers) showed a difference of up to 9 years old with each other. It is well known that $\mathrm{FEV}_{1}$ and FVC decrease with age, raising a concern to compare directly these groups in which the mean age differs. This concern is particularly important in case that values of $\mathrm{FEV}_{1}$ and $\mathrm{FVC}$ in some group are lower than those in the other group when the ages in the former group are older than the latter's. In the present study, however, the spirometric measures were lower in current smokers than in never smokers, despite that the current smokers were younger than the never smokers, which keeps logical relevance in negative impacts of current smoking on spirometric measures. Additionally, the low $\% \mathrm{FVC}$, less than $80 \%$, in the male current smokers was unexpected in this study. We have used, for the first time, the reference values for spirometric measures that were derived from Japanese criteria updated on 2014. There might be some regional het- erogeneity in relationships between anthropometric measures and spirometric measures, at least in males. Next, a small sample size of female current smokers and former smokers makes it unfeasible to provide the comprehensive understanding for the relationship between potential airflow limitation and smoking. Finally, the study did not investigate the impact of environmental cigarette smoking on the prevalence of airflow limitation in never smokers. Increasing exposure to environmental cigarette smoking is associated with increasing prevalence of respiratory symptoms that are common in COPD (Janson et al. 2001; Larsson et al. 2003; Yin et al. 2007). Many of never smokers with potential airflow limitation were females. Given that a majority of female never smokers had lived as housewives in Hisayama, partner's smoking might affect their pulmonary function.

In summary, the prevalence of airflow limitation defined by pre-bronchodilator $\mathrm{FEV}_{1} / \mathrm{FVC}<70 \%$ was $14.6 \%$ in males and $13.7 \%$ in females, and the prevalence of post-bronchodilator airway limitation was $8.7 \%$ and $8.7 \%$, respectively. These results suggest that post-bronchodilator spirometry in health checkup settings would reduce the number of subjects with probable COPD to twothird and then reduce the default evaluation.

\section{Acknowledgments}

This study was supported by Health and Labor Sciences Research Grants from the Ministry of Health, Labour and Welfare of Japan. We thank Ms. Ayako Hashizume and Ms. Chiaki Yahiro for their technical assistance.

\section{The Hisayama Pulmonary Physiology Study Group}

Satoru Fukuyama, Koichiro Matsumoto, Takako Nakano, Atsushi Moriwaki, Keiko Kan-o, Yuko Matsunaga, Naotaka Noda, Yukari Tajiri-Asai, Hiroko Hirai, Yumiko Ishii, Saaka Hamano, Nanae Seki, Ken Tonai, Kunihiro Kudo, Kentaro Tamura, Miyoko Tatsuta, Hiroki Shundo, Aimi Enokizu, Yoichi Nakanishi (Research Institute for Diseases of the Chest, Graduate School of Medical Sciences, Kyushu University, Fukuoka, Japan); Go Tsukuya, Takuya Samukawa, Takako Inoue, Hiromasa Inoue (Department of Pulmonary Medicine, Graduate School of Medical and Dental Sciences, Kagoshima University, Kagoshima, Japan); Makoto Yoshida, Masashi Komori, Yasuko Kaneko, Tomoaki Iwanaga (Division of Respiratory Medicine, National Hospital Organization Fukuoka Hospital, Fukuoka, Japan); Shohei Takata, Mikiko Matsuo (Division of Respiratory Medicine, National Hospital Organization Fukuoka-Higashi Medical Center, Fukuoka, Japan); Hiroshi Koto (Division of Respiratory Medicine, Kyushu Central Hospital of the Mutual Aid Association of Public School Teachers, Fukuoka, Japan).

\section{Author Contributions}

S.F., K.M., and H.I. designed the study, collected data, 
and wrote the initial drafts of manuscript. K.K., N.N., Y. T.-A., T.N., and Y.I. collected data. Y.K. (Kaneko) constructed the database. Y.K. (Kiyohara) and Y.N. advised the designing of study. All authors read and approved the final manuscript.

\section{Conflict of Interest}

The authors declare no conflict of interest.

\section{References}

Bustacchini, S., Chiatti, C., Furneri, G., Lattanzio, F. \& Mantovani, L.G. (2011) The economic burden of chronic obstructive pulmonary disease in the elderly: results from a systematic review of the literature. Curr. Opin. Pulm. Med., 17 Suppl 1, S35-S41.

Fukuchi, Y., Nishimura, M., Ichinose, M., Adachi, M., Nagai, A., Kuriyama, T., Takahashi, K., Nishimura, K., Ishioka, S., Aizawa, H. \& Zaher, C. (2004) COPD in Japan: the Nippon COPD Epidemiology study. Respirology, 9, 458-465.

Global Initiative for Chronic Obstructive Lung Disease (GOLD) (2014) Global strategy for the diagnosis, management and prevention of COPD.

[Updated: January, 2014].

http://www.goldcopd.com [Accessed: January 23, 2014].

Hata, J., Ninomiya, T., Hirakawa, Y., Nagata, M., Mukai, N., Gotoh, S., Fukuhara, M., Ikeda, F., Shikata, K., Yoshida, D., Yonemoto, K., Kamouchi, M., Kitazono, T. \& Kiyohara, Y. (2013) Secular trends in cardiovascular disease and its risk factors in Japanese: half-century data from the Hisayama Study (1961-2009). Circulation, 128, 1198-1205.

Janson, C., Chinn, S., Jarvis, D., Zock, J.P., Toren, K. \& Burney, P.; the European Community Respiratory Health Survey (2001) Effect of passive smoking on respiratory symptoms, bronchial responsiveness, lung function, and total serum $\operatorname{IgE}$ in the European Community Respiratory Health Survey: a crosssectional study. Lancet, 358, 2103-2109.

Kohansal, R., Martinez-Camblor, P., Agusti, A., Buist, A.S., Mannino, D.M. \& Soriano, J.B. (2009) The natural history of chronic airflow obstruction revisited: an analysis of the Framingham Offspring Cohort. Am. J. Respir. Crit. Care Med., 180, 3-10.

Kojima, S., Sakakibara, H., Motani, S., Hirose, K., Mizuno, F., Ochiai, M. \& Hashimoto, S. (2007) Incidence of chronic obstructive pulmonary disease, and the relationship between age and smoking in a Japanese population. J. Epidemiol., 17, 54-60.

Kubota, M., Kobayashi, H., Quanjer, P.H., Omori, H., Tatsumi, K. \& Kanazawa, M.; the Clinical Pulmonary Functions Committee of the Japanese Respiratory Society (2014) Reference values for spirometry, including vital capacity, in Japanese adults calculated with the LMS method and compared with previous values. Respir. Investig., 62, 242-250.

Larsson, M.L., Loit, H.M., Meren, M., Põlluste, J., Magnusson, A., Larsson, K. \& Lundbäck, B. (2003) Passive smoking and respiratory symptoms in the FinEsS Study. Eur. Respir. J., 21, 672-676.

Matsumoto, K., Seki, N., Fukuyama, S., Moriwaki, A., Kan-o, K., Matsunaga, Y., Noda, N., Yoshida, M., Koto, H., Takata, S., Nakanishi, Y., Kiyohara, Y. \& Inoue, H. (2015) Prevalence of asthma with airflow limitation, COPD, and COPD with variable airflow limitation in older subjects in a general Japanese population: the Hisayama Study. Respir. Investig., 53, 22-29.

Pellegrino, R., Viegi, G., Brusasco, V., Crapo, R.O., Burgos, F., Casaburi, R., Coates, A., van der Grinten, C.P., Gustafsson, P., Hankinson, J., Jensen, R., Johnson, D.C., MacIntyre, N., McKay, R., Miller, M.R., et al. (2005) Interpretative strategies for lung function tests. Eur. Repir. J., 26, 948-968.

Scholes, S., Moody, A. \& Mindell, J.S. (2014) Estimating population prevalence of potential airflow obstruction using different spirometric criteria: a pooled cross-sectional analysis of persons aged 40-95 years in England and Wales. BMJ Open, 4, e005685.

The Japanese Respiratory Society (2004) Guidelines of respiratory function tests: spirometry, flow-volume curve, diffusion capacity of the lung. Nihon Kokyuki Gakkai Zassi, Suppl, 1-56 (in Japanese).

The Statistics Bureau of Japan (2010) Japan Census. http://www.stat.go.jp/english/data/kokusei/pdf/20111026.pdf [Accessed: October 26, 2011].

Tilert, T., Dillon, C., Paulose-Ram, R., Hnizdo, E. \& Doney, B. (2013) Estimating the U.S. prevalence of chronic obstructive pulmonary disease using pre- and post-bronchodilator spirometry: the National Health and Nutrition Examination Survey (NHANES) 2007-2010. Respir. Res., 14, 103.

Toyoda, K. (2013) Epidemiology and registry studies of stroke in Japan. J. Stroke, 15, 21-26.

Yin, P., Jiang, C.Q., Cheng, K.K., Lam, T.H., Lam, K.H., Miller, M.R., Zhang, W.S., Thomas, G.N. \& Adab, P. (2007) Passive smoking exposure and risk of COPD among adults in China: the Guangzhou Biobank Cohort Study. Lancet, 370, 751-757. 